\title{
Quaghebeur, Joëlle, Soleil, Sylvain (dir.),Le pouvoir et la foi au Moyen Âge en Bretagne et dans l'Europe de l'Ouest
}

Nolwenn Lachater

\section{(2) OpenEdition \\ Journals}

Édition électronique

URL : http://journals.openedition.org/abpo/2024

DOI : $10.4000 / a b p o .2024$

ISSN : 2108-6443

Éditeur

Presses universitaires de Rennes

Édition imprimée

Date de publication : 30 mai 2011

Pagination : 175-177

ISBN : 978-2-7535-1691-5

ISSN : 0399-0826

Référence électronique

Nolwenn Lachater, « Quaghebeur, Joëlle, Soleil, Sylvain (dir.), Le pouvoir et la foi au Moyen Âge en Bretagne et dans l'Europe de l'Ouest », Annales de Bretagne et des Pays de l'Ouest [En ligne], 118-2 I 2011, mis en ligne le 30 juin 2013, consulté le 23 septembre 2020. URL : http:// journals.openedition.org/abpo/2024 ; DOI : https://doi.org/10.4000/abpo.2024

Ce document a été généré automatiquement le 23 septembre 2020.

(c) Presses universitaires de Rennes 


\title{
Quaghebeur, Joëlle, Soleil, Sylvain (dir.),Le pouvoir et la foi au Moyen Âge en Bretagne et dans l'Europe de l'Ouest
}

\author{
Nolwenn Lachater
}

\section{RÉFÉRENCE}

QUAGHEBeUR, Joëlle, SoleIL, Sylvain (dir.), Le pouvoir et la foi au Moyen Âge en Bretagne et dans l'Europe de l'Ouest, Rennes, PUR, coll. « Histoire », Britannia Monastica 13-14, 2010, $750 \mathrm{p} ., 35 €$.

1 Ce volumineux recueil de Mélanges à la mémoire du professeur Hubert Guillotel, ne regroupe pas moins de quarante cinq contributions. Le CIRDoMoC (Centre International de Recherches et de Documentation sur le Monachisme Celtique - Landévennec) dont il contribuait assidument à animer les journées d'études depuis la fondation en 1988 et le Centre d'Histoire du Droit de l'Université de Rennes 1 où il a assuré son enseignement et poursuivi ses recherches de 1999 à son décès prématuré en juin 2004 se sont associés pour publier dans la collection "Histoire» des Presses Universitaires de Rennes cet hommage collectif qui se veut "l'écho de la vie et de l'œuvre de celui qu'il entend honorer ", comme l'écrit le professeur Olivier Guyot en introduction à ce livre. La «Liste des travaux et publications de Hubert Guillotel» qui clôture l'ouvrage atteste de la fécondité des recherches de celui-ci. Plusieurs contributeurs ne manquent pas de rappeler avec quelle rigueur il laissait attendre ses textes jusqu'à ce qu'il les estime arrivés à maturité (et donc dignes d'être publiés).

2 «Pouvoir» et « Foi », les deux axes de réflexion autour desquels les coordonateurs de ces Mélanges, Joëlle Quaghebeur (maître de conférences à l'université de Bretagne SudLorient et secrétaire du CIRDoMoC) et Sylvain Soleil (professeur à l'université Rennes 1 
et directeur jusqu'en 2010 du Centre d'Histoire du Droit - CHD), ont articulé l'ouvrage reprennent les orientations dominantes de l'œuvre d'Hubert Guillotel. Celui-ci ne concevait pas l'histoire de la Bretagne médiévale, de ses hommes et de ses institutions comme étroitement repliée sur elle-même mais au contraire au cœur des réalités et innovations qu'ont connues alors la France et l'Europe. Les comparaisons avec des espaces géographiques différents permettent de déterminer spécificités ou ressemblances avec le monde breton. Nombre des contributions rassemblées ici font appel à l'archéologie, la toponymie, l'onomastique ou la linguistique attestant de l'ouverture d'Hubert Guillotel à l'interdisciplinarité.

3 Au vu du nombre d'articles, ce compte rendu ne peut naturellement pas s'attarder sur chacune de ces monographies, ce qui n'est d'ailleurs pas son objectif. La foi due à Dieu, jurée au prince ou promise à son seigneur fonde l'encadrement des âmes et le pouvoir sur les hommes intimement liés, l'un à l'autre. La première partie de ce livre traite du pouvoir sur les âmes décliné en trois aspects : « prier », " réformer » et " écrire ». Dans l'Ouest, l'un des établissements majeurs de la réforme monastique carolingienne se trouve être le monastère de Glanfeuil (Saint-Maur-sur-Loire), en contact avec l'abbaye de Redon. Guy Jarousseau ( Jus proprietarium et jus ecclesiasticum. La restauration de l'abbaye de Saint-Maur-sur-Loire au milieu du $\mathrm{IX}^{\mathrm{e}}$ siècle ») montre l'évolution institutionnelle de celle-ci grâce à une série d'actes par lesquels le pouvoir royal restaure cet établissement. La réforme bénédictine de grandes abbayes comme Glanfeuil ou Redon entraîne celle de ses établissements monastiques secondaires dépendants dont le nombre augmente selon Bernard Tanguy ( $"$ Monateriola au $\mathrm{Ix}^{\mathrm{e}}$ et $\mathrm{X}^{\mathrm{e}}$ siècle, d'après le cartulaire de Saint-Sauveur de Redon et les Gesta des saints de Redon »). Cette restauration apparaît significative d'un fort investissement des lignages nobles dans l'Église. Ultérieurement, les abbés cisterciens, d'après les recherches initiées par Véronique Gazeau ( "Jalons pour une enquête prosopographique : les abbés cisterciens de Normandie») appartiennent à cette haute sphère sociale, identifiable dans les cartulaires et les sources nécrologiques. La réforme grégorienne semble intervenir précocement dans l'Ouest comme le montre Daniel Pichot ( « La notice $294 \mathrm{du}$ Cartulaire de Redon : les moines, la réforme et la société »). L'arrivée de ce monachisme réformé s'associe donc au pouvoir laïc et aussi naturellement au pouvoir religieux. Certains prélats font figure de modèles de réformateurs comme saint Gilduin de Dol décrit par Michel Debary («Saint Guilduin et le Pape Grégoire VII »). La mise en place de cette réforme ne se fit donc pas sans difficulté ni arrière-pensées. Dans la Bretagne ducale, André-Yves Bourgès explique comment les ducs de Cornouaille ont favorisé et imposé la réforme grégorienne pour renforcer leur pouvoir au détriment de leurs barons ("Propagande ducale, réforme grégorienne et renouveau monastique: la production hagiographique en Bretagne sous les ducs de la maison de Cornouaille »). Pour légitimer leur place à la tête du duché, les ducs ont d'ailleurs développé toute une littérature hagiographique comme moyen de propagande. La maîtrise de l'écrit et son utilisation par les clercs réformateurs constitue donc également une question prenante. L'étude minutieuse des sources diplomatiques médiévales mérite critique et méfiance, car les problèmes sont nombreux: "C'est un faux!», s'exclamait fréquemment $\mathrm{H}$. Guillotel (Ph. Guigon, «La donnation d'Enesmur à l'abbaye Saint-Sauveur de Redon »). Aussi, l'origine de ces actes, la date, le pourquoi et le comment de leur rédaction, doivent-ils retenir l'attention (Armelle Le Huerou, «L'archiepiscopus Dolensis au début du XII ${ }^{e}$ siècle. Esquisse d'un catalogue des actes de l'archevêque Baudri (1107-1130) »)... 
4 Autour de la thématique « Le pouvoir sur les hommes : juger, gouverner et combattre ", la seconde partie de ces Mélanges risquerait davantage de donner - de prime abord l'impression d'un « patchwork » qui irait du baptême de Clovis (H. Oudart, «Ut omnes te ament et timeant: Clovis protecteur des faibles et anti-tyran dans la première lettre au roi de l'évêque Rémi ?») à la Révolution (S. Soleil, «La Bretagne nationalisée. Droit et idéologie dans le débat parlementaire des 8-11 janvier $1789 »)$. Les éditeurs ont réussi à éviter cet écueil en articulant les diverses contributions sur les notions de jugement, de gouvernement et de combat, si bien que les perspectives offertes par les prolongements sur l'époque moderne et contemporaine, présentées notamment par ses collègues juristes, répondent au souci de lire l'histoire sur la longue durée qui était celui de Hubert Guillotel. On ne pointera ici que quelques titres pour illustrer ce propos. C'est ainsi que Soazick Kerneis ( «Jugement des hommes ; jugement de Dieu. Les Irlandais et la vérité judiciaire. $\mathrm{v}^{\mathrm{e}}-\mathrm{VIII}^{\mathrm{e}}$ siècles ») analyse finement l'ordalie comme un instrument judiciaire qui participe de la sacralisation de la royauté carolingienne inspirée par les lettrés irlandais. Marie-Yvonne Crépin (« Un procès criminel devant la juridiction de l'abbaye de la Vieuville ») choisit de mener une étude de cas sur le dossier d'un crime d'inceste et d'infanticide instruit en 1763 par la juridiction seigneuriale de l'abbaye de la Vieuville à laquelle Hubert Guillotel était particulièrement attaché tandis qu'Alain Berbouche («La seigneurie ecclésiastique de Saint-Malo à la fin de l'Ancien Régime ») étudie le fonctionnement de la justice ecclésiastique malouine jusqu'à son abrogation à l'issue de la Nuit du 4 août et son remplacement par le tribunal civil et correctionnel de Saint-Malo en 1790.

5 Ce dernier article s'inscrit parmi les études consacrées aux questions de gouvernance (pour recourir à un néologisme à la mode!). En effet, comme pour «juger», "gouverner" nécessite des lieux, des hommes et des procédures. André Chédeville, décédé en juin 2010, peu avant la sortie de ces Mélanges, a tenu à exprimer son amitié pour Hubert Guillotel en lui dédiant un commentaire lumineux du rite de la plantation du pal dans la fausse charte de fondation du château de Josselin (« Figens palum in castello edificando, ut mos est... À propos de la fondation du château de Josselin »). Selon Christiane Plessix-Buisset («Piété et charité dans la Très-ancienne Coutume de Bretagne »), ce «traité de droit et de procédure civile et pénale » élaboré au début du $\mathrm{XIV}^{\mathrm{e}}$ siècle apparaît aussi comme une sorte de catéchisme et de livre de morale à destination des gouvernants et des praticiens en s'inspirant des préceptes de la charité chrétienne pour défendre les causes justes, protéger les pauvres et prôner la loyauté et la vertu. La contribution de Jean-Pierre Brancourt, («Esprit de la légitimité sous le règne de Charles VII ») selon laquelle les éléments de la légitimité à la couronne de France n'acquièrent de portée juridique définitive que sous Charles VII - avec entre autre la masculinité et l'indisponibilité de la couronne - fait écho, à l'article de Franck Bouscau dans la sous-partie précédente («Réflexions sur les aspects juridiques de la succession à la couronne de Bretagne ») qui montre en quoi la mise en place de ce règles successorales à partir du contexte de l'affrontement entre Français et Anglais finit par différer sur certains points du droit coutumier et féodal. La question de la «marche», revisitée aujourd'hui à nouveaux frais par les historiens, sous-tend plusieurs contributions de la dernière partie qui traite de la thématique du combat. Entre autres, selon Eric van Torhoudt («La résistance franco-bretonne à l'expansion normande dans le nord-ouest de la Neustrie (924-954) : une marche de Normandie») l'association des Bretons et des Francs d'Hugues le Grand aurair permis au duché de repousser $\mathrm{au} \mathrm{x}^{\mathrm{e}}$ siècle les attaques normandes tout en participant à la politique 
d'endiguement des Normands voulue par le dux Francorum. Quant à l'étude de JeanClaude Meuret ( $\mathrm{Au}$ cœur de la marche bretonne, l'ascension et le démantèlement d'une grande châtellenie, La Guerche-Pouancé: milieu XI ${ }^{\mathrm{e}}$-milieu XIII ${ }^{\mathrm{e}}$ siècle ») en démêlant l'écheveau du lignage châtelain de la Guerche, elle outrepasse l'échelon "monographique et micro-régional» pour mettre en évidence un "changement d'échelle politique, économique et humaine » qui se développe au contact de la France dans cette zone de Marche.

En définitive, Pouvoir et foi sont bien indissociables au Moyen Âge, en Bretagne comme dans tout l'Occident ainsi que le montrent les thèmes englobant ces deux sujets qui s'entrecroisent maintes fois dans ces Mélanges, dans la droite ligne des études et des travaux réalisés sur la Bretagne d'Hubert Guillotel. En étayant les réflexions par de l'archéologie, de la toponymie, de l'onomastique ou de la linguistique, et en élargissant ces perspectives pluridisciplinaires dans l'espace et dans le temps, les recherches se font plus sûres, plus pertinentes, en un mot plus intéressantes.

\section{INDEX}

Thèmes : Bretagne

Index chronologique : Moyen Âge

\section{AUTEURS}

\section{NOLWENN LACHATER}

Master HSC - Université de Rennes 2 Haute-Bretagne 